\title{
Light WIMP Searches Involving Electron Scattering
}

\author{
J. D. Vergados $\mathbb{D}^{1,2,3,4}$ Ch. C. Moustakidis $\left(D,{ }^{5}\right.$ Yeuk-Kwan E. Cheung, ${ }^{4}$ H. Ejiri $\left(\mathbb{D},{ }^{6}\right.$ \\ Yeongduk Kim, ${ }^{7}$ and Jeong-Yeon Lee ${ }^{7}$ \\ ${ }^{1}$ TEI of Western Macedonia, 50100 Kozani, Greece \\ ${ }^{2}$ CAPP, IBS, Yuseong, Daejeon 34144, Republic of Korea \\ ${ }^{3}$ CoEPP and Centre for the Subatomic Structure of Matter (CSSM), University of Adelaide, Adelaide, SA 5005, Australia \\ ${ }^{4}$ Department of Physics, Nanjing University, 22 Hankou Road, Nanjing 210093, China \\ ${ }^{5}$ Department of Theoretical Physics, Aristotle University of Thessaloniki, 54124 Thessaloniki, Greece \\ ${ }^{6}$ RCNP, Osaka University, Osaka 567-0047, Japan \\ ${ }^{7}$ Center for Underground Physics, IBS, Daejeon 34074, Republic of Korea
}

Correspondence should be addressed to Ch. C. Moustakidis; moustaki@auth.gr

Received 20 November 2017; Accepted 8 March 2018; Published 15 April 2018

Academic Editor: Filipe R. Joaquim

Copyright (C) 2018 J. D. Vergados et al. This is an open access article distributed under the Creative Commons Attribution License, which permits unrestricted use, distribution, and reproduction in any medium, provided the original work is properly cited. The publication of this article was funded by $\mathrm{SCOAP}^{3}$.

\begin{abstract}
In the present work we examine the possibility of detecting electrons in light dark matter searches. These detectors are considered to be the most appropriate for detecting dark matter particles with a mass in the $\mathrm{MeV}$ region. We analyze theoretically some key issues involved in such detection. More specifically we consider a particle model involving WIMPs interacting with fermions via $Z$-exchange. We find that for WIMPs with mass in the electron mass range the cross section for WIMP-atomic electron scattering is affected by the electron binding. For WIMPs more than 20 times heavier than the electron, the binding affects the kinematics very little. As a result, many electrons can be ejected with energy which increases linearly with the WIMP mass, but the cross section is somewhat reduced depending on the bound state wave function employed. On the other hand for lighter WIMPs, the effect of binding is dramatic. More specifically at most 10 electrons, namely, those with binding energy below $10 \mathrm{eV}$, become available even in the case of WIMPs with a mass as large as 20 times the electron mass. Even fewer electrons contribute if the WIMPs are lighter. The cross section is, however, substantially enhanced by the Fermi function corrections, which become more important at low energies of the outgoing electrons. Thus events of 0.5-2.5 per kg-y become possible.
\end{abstract}

\section{Introduction}

The combined MAXIMA-1 [1-3], BOOMERANG [4, 5] DASI [6], and COBE/DMR Cosmic Microwave Background (CMB) observations [7] imply that the universe is flat [8] and that most of the matter in the universe is dark [9], that is, exotic. These results have been confirmed and improved by the recent WMAP [10] and Planck [11] data. Combining the data of these quite precise measurements one finds

$$
\begin{aligned}
\Omega_{b} & =0.0456 \pm 0.0015, \\
\Omega_{\mathrm{CDM}} & =0.228 \pm 0.013, \\
\Omega_{\Lambda} & =0.726 \pm 0.015 ;
\end{aligned}
$$

(the more recent Planck data yield a slightly different combination, $\Omega_{\mathrm{CDM}}=0.274 \pm 0.020, \Omega_{\Lambda}=0.686 \pm 0.020$ ). It is worth mentioning that both the WMAP and the Plank observations yield essentially the same value of $\Omega_{m} h^{2}$, but they differ in the value of $h$, namely, $h=0.704 \pm 0.013$ (WMAP) and $h=0.673 \pm 0.012$ (Planck). Since any "invisible" nonexotic component cannot possibly exceed $40 \%$ of the above $\Omega_{\mathrm{CDM}}$ [12], exotic (nonbaryonic) matter is required and there is room for cold dark matter candidates or WIMPs (Weakly Interacting Massive Particles).

Even though there exists firm indirect evidence for a halo of dark matter in galaxies from the observed rotational curves, see, for example, the review [13], it is essential to directly detect such matter in order to unravel the nature 
of the constituents of dark matter. The possibility of such detection, however, depends on the nature of the dark matter constituents and their interactions.

The WIMPs are expected to have a velocity distribution with an average velocity which is close to the rotational velocity $v_{0}$ of the Sun around the galaxy; that is, they are completely nonrelativistic. In fact a Maxwell-Boltzmann leads to a maximum energy transfer which is close to the average WIMP kinetic energy $\prec T>\approx 0.4 \times 10^{-6} \mathrm{mc}^{2}$. Thus for $\mathrm{GeV}$ WIMPs this average is in the $\mathrm{keV}$ regime, not high enough to excite the nucleus, but sufficient to measure the nuclear recoil energy. For light dark matter particles in the $\mathrm{MeV}$ region, which we will also call WIMPs, the average energy that can be transferred is in the few $\mathrm{eV}$ regions. So this light WIMPs can be detected by measuring the electron recoil after the collision. Electrons may of course be produced by heavy WIMPs after they collide with a heavy target which results in a shakeup of the atom yielding "primordial" electron production [14-16]. This approach for sufficiently heavy WIMPs and target nuclei can produce electrons energies even in the $30 \mathrm{keV}$ region, with a spectrum very different from that arising after a direct WIMP-electron collision. Furthermore WIMP-electron collisions involving WIMPs with masses in the few $\mathrm{GeV}$ region have also recently appeared [17, 18]. In the present work, however, we will restrict ourselves in the case of light WIMPs with a mass in the region of the electron mass.

We will draw from the experience involving WIMPs in the $\mathrm{GeV}$ region. The event rate for such a process can be computed from the following ingredients [19]: (i) the elementary WIMP-electron cross section. The total Higgs decay width $[20] \Gamma=6.1_{-2.9}^{7.7} \mathrm{MeV}$ determined by the LHC data imposes severe constraints on the WIMP-fermion interactions mediated with Higgs exchange. Thus we will consider the case of a WIMP which interacts with fermions via $Z$-exchange. In this case we will relate the needed WIMP-electron cross to the isovector spin dependent WIMPnucleon cross and exploit the information available for the latter. (ii) The knowledge of the WIMP particle density in our vicinity: this is extracted from WIMP density in the neighborhood of the solar system, obtained from the rotation curves measurements. The number density of these $\mathrm{MeV}$ WIMPs, however, is expected to be six orders of magnitude bigger than that of the standard WIMPs due to the smaller WIMP mass involved. (iii) The WIMP velocity distribution: in the present work we will consider a Maxwell-Boltzmann (MB) distribution.

In the electron recoil experiments, like the nuclear measurements first proposed more than 30 years ago [21], one has to face the problem that the process of interest does not have a characteristic feature to distinguish it from the background. So since low counting rates are expected the background is a formidable problem. Some special features of the WIMP interaction can be exploited to reduce the background problems, such as the modulation effect: this yields a periodic signal due to the motion of the Earth around the Sun. Unfortunately this effect, also proposed a long time ago [22] and subsequently studied by many authors [19, 23-31], is small in the case of nuclear recoils, but we expect it to be a bit larger in the case of the electron recoils. There has always been an interest in light WIMPs, see, for example, the recent work [32]. In fact the first direct detection limits on sub-GeV dark matter from XENON10 have recently been obtained [33]. This is encouraging, but based on our experience with standard nuclear recoil experiments to excited states [34], one has to make sure that the proper kinematics has to be used in dealing with bound electrons. Clearly the binding electron energy plays a similar role as the excitation energy of the nucleus, in determining the small fraction of the WIMP's energy to be transferred to the recoiling system. It is therefore clear that light WIMPs are quite different in energy, mass, interacting particle, and flux. Accordingly one needs detectors capable of detecting low energy light WIMPs in the midst of formidable backgrounds, that is, detectors which are completely different from current WIMP detectors employed for heavy WIMP searches.

\section{The Particle Model}

The narrow decay width of the Higgs boson obtained with LHC data imposes severe constraints on the Higgs mediated WIMP-SM fermion interaction. It may thus be more favorable to look for $Z$ - boson mediated interactions, which have so far been considered only in the hadron spin induced dark matter searches. One finds that the SM coupling of the $Z$ to the electron takes the form $\bar{e} \gamma_{\lambda}\left(1-2 \sin _{\theta_{W}}^{2}-\gamma_{5}\right) e \approx$ $-\bar{e} \gamma_{\lambda} \gamma_{5} e$, where $\theta_{W}$ is the Weiberg angle. On the other hand the corresponding isovector quark interaction, expected to dominate in the scattering, takes the form $-\bar{q} \tau_{3} \gamma_{\lambda} \gamma_{5} q$, which leads to $-g_{A} \bar{N} \tau_{3} \gamma_{\lambda} \gamma_{5} N$ at the nucleon level. It is therefore natural to relate, if possible, the elementary WIMP-electron cross section to that of the spin induced isovector elementary WIMP-nucleon interaction, since for the latter there exists more theoretical and experimental information

Indeed if both the WIMP $\chi$ and the target with mass $m$ are nonrelativistic particles the cross section can be cast in the form

$$
\begin{aligned}
d \sigma= & \frac{1}{v} \mathscr{M}^{2} \frac{1}{(2 \pi)^{2}} d^{3} \mathbf{p}_{\chi}^{\prime} d^{3} \mathbf{q} \delta\left(\mathbf{p}_{\chi}-\mathbf{p}_{\chi}^{\prime}-\mathbf{q}\right) \\
& \cdot \delta\left(\frac{\mathbf{p}_{\chi}^{2}}{2 m_{\chi}}-\frac{\mathbf{p}_{\chi}^{\prime 2}}{2 m_{\chi}}-\frac{\mathbf{q}^{2}}{2 m}\right) .
\end{aligned}
$$

Integrating over the momenta we find

$$
d \sigma=\frac{1}{v} \mathscr{M}^{2} \frac{1}{(2 \pi)^{2}} 2 \pi q^{2} d q d \xi \delta\left(q v \xi-\frac{q^{2}}{2 \mu_{r}}\right),
$$

where

$$
\frac{1}{\mu_{r}}=\frac{1}{m_{\chi}}+\frac{1}{m}, \quad \mu_{r}=\frac{m_{e} m_{\chi}}{m_{e}+m_{\chi}}=\text { reduced mass }
$$

with $\xi=\widehat{p}_{\chi} \cdot \widehat{q} \geq 0$. Thus

$$
d \sigma=\frac{1}{v} \mathscr{M}^{2} \frac{1}{2 \pi} d \xi \frac{\left(2 \mu_{r} v \xi\right)^{2}}{|v \xi|}=\frac{1}{\pi} \mathscr{M}^{2} \mu_{r}^{2} 2 \xi d \xi .
$$


To this end for the elementary isovector proton cross section we write

$$
\sigma_{1}=\Lambda\left(1+\frac{m_{p}}{m_{\chi}}\right)^{-2} m_{p}^{2},
$$

where $\Lambda$ is a constant. Before proceeding further let us examine available information on $\sigma_{1}$ [35], which is summarized here: (a) supersymmetric models in which the LSP (lightest supersymmetric particle) communicates with the quarks via the exchange of the $Z$-boson [36]. In this case in the constrained minimal supersymmetric standard model one finds sizable spin induced cross sections [37, 38], $\sigma_{p}$ and $\sigma_{n}$ as large as $10^{-3} \mathrm{pb}$ (the proton neutron representation was chosen, since the experiments are analyzed this way). If, however, the isoscalar contribution is negligible the proton and the neutron amplitudes are opposite and $\sigma_{n} \approx \sigma_{p} \approx \sigma_{1}$. (b) Kaluza-Klein theories in models with Universal Extra Dimensions [39] in which the dark matter is a heavy neutrino communicating with matter via $Z$-exchange. In such models the couplings are of the order of the standard model couplings and one finds $\sigma_{1}=(1 / 32 \pi)\left(G_{F} m_{p}\right)^{2} \approx 5 \times 10^{-4} \mathrm{pb}$. (c) The WIMP is a spin $3 / 2$ particle [40]. In this case only the isovector contribution exists, leading to $\sigma_{1}(N) \approx 1.7 \times$ $10^{-38} \mathrm{~cm}^{-2}=1.7 \times 10^{-2} \mathrm{pb}$. (d) Experimental limits: there exist some, namely, for ${ }^{129} \mathrm{Xe}$ and ${ }^{131} \mathrm{Xe}[41]$ and ${ }^{19} \mathrm{~F}[42-$ 46]. From the Xe data a limit is extracted on the elementary neutron SD cross section of $\sigma_{n}=2 \times 10^{-40} \mathrm{~cm}^{2}=2 \times$ $10^{-4} \mathrm{pb}$ and $\sigma_{p}=2 \times 10^{-38} \mathrm{~cm}^{2}=2.0 \times 10^{-2} \mathrm{pb}$ for the proton SD cross section, while from the ${ }^{19} \mathrm{~F}$ target a slightly smaller limit is extracted on the proton SD cross section, $\sigma_{p}=1 \times 10^{-38} \mathrm{~cm}^{2}=1.0 \times 10^{-2} \mathrm{pb}$. These limits were based on nuclear physics considerations, namely, the nuclear spin matrix elements in the proton neutron representation. This explains the difference of the two limits extracted from the Xe data. For illustration purposes we will adopt the value [35] $\sigma_{1} \approx 1.7 \times 10^{-2} \mathrm{pb}$. With this value of $\sigma_{1}$ we find that the parameter relevant for electron scattering is

$$
\sigma_{0}=\sigma_{1} \frac{m_{e}^{2}}{m_{p}^{2}} \approx 5.0 \times 10^{-9} \mathrm{pb} .
$$

\section{Experimental Aspects}

Light WIMPs are quite different in energy, mass, interacting particle, and flux. Accordingly one needs detectors which are completely different from current WIMP detectors for heavy WIMPs. Detectors are required to observe low energy light WIMP signals beyond/among BG signals to identify the light WIMPs. Experimental aspects to be considered for light WIMP detectors are: (i) the particle to be detected, (ii) the event rate, (iii) the signal pulse height, (iv) the background rate, and $(v)$ the detector threshold energy. We will now examine each of these items.

(1) Particle to Be Detected. Light WIMPs are detected by observing a recoil/scattered electron in the continuum region. In case that the WIMP interaction produces an ionelectron pair, one can detect the ion and/or the electron, and/or photons associated with the ion-electron pair. If the recoil electron or the ion-electron pair energy is absorbed by the detector, one may measure the temperature change. These are similar to those from heavy WIMPs except that their energies are very different. It is noted that atomic bound electrons are not excited by the light WIMPs with $E \leq$ a few $\mathrm{eV}$.

(2) Event Rate. The cross section of $\sigma_{0} \approx 5.0 \times 10^{-9} \mathrm{pb}$ is an order of magnitude larger than the present XENON limit of $\sigma_{0} \approx 10^{-9} \mathrm{pb}$ for heavy WIMPs [47]. The flux rate is around $n \approx 0.8 \times 10^{10} \mathrm{~cm}^{-2} \mathrm{~s}^{-1}$, which is larger by a factor of $50 \mathrm{GeV} / 0.5 \mathrm{MeV} \approx 10^{5}$. Then the event rate for Xe detector is around $R \approx 1.1 \times 10^{3} /(\mathrm{t} \cdot \mathrm{y})$, which is also a bit larger than the present limit for $50 \mathrm{GeV}$ heavy WIMPs [47].

(3) Signal Pulse Height. The electron signal energy for light WIMPs is around $0.5-1 \mathrm{eV}$. This energy is 4 orders of magnitude smaller than the Xe nuclear recoil energy of around $25 \mathrm{keV}$ for the $50 \mathrm{GeV}$ WIMP. The nuclear recoil signal is quenched by a factor 2-20, depending on the atomic number, in most heavy WIMP detectors. Thus the actual signal height for the light WIMP is 3 orders of magnitude smaller than that for the heavy WIMP.

(4) Background Rate. There are three types of background origins for WIMP detectors, radioactive (RI) impurities, neutrons associated with cosmic rays, and electric noises. $\beta-\gamma$ rays from RI impurities produce BG electron signals, which are similar to electron signals from light WIMPs as well as those encountered in the case of double $\beta$ decay detectors, which measure $\beta$ rays. BG rate for a typical future DBD (double $\beta$ decay) detectors is around $1 /(\mathrm{t} y \mathrm{keV})=10^{-3} / \mathrm{t}$ $\mathrm{y} \mathrm{eV}$ ) at a few $\mathrm{MeV}$ regions [48]. Then one may expect a similar BG rate in the $\mathrm{eV}$ region. This is 3 orders of magnitude smaller than the signal rate. Neutrons do not contribute to BGs in light WIMP detectors, although nuclear recoils from neutron nuclear reactions are most serious BGs for heavy WIMP detectors. Electric noises are most serious for light WIMP detectors because of the very low energy signals. The nuclear recoil energy from heavy WIMPs is typically a few $10 \mathrm{keV}$, and the signal pulse height is around a few $\mathrm{keV}$ if they are quenched, depending on the detector. This is of the same order of magnitude as electric noise levels. Thus one can search for heavy WIMPs by measuring the higher velocity component above the electric noises On the other hand, the signal height for light WIMP is far below that of typical electric noises for current heavy WIMP detector.

(5) Energy Threshold. The energy threshold $E_{\text {th }}$ for WIMP detectors is set necessarily below the WIMP signal, but just above the electric noise to be free from the noise. Then a very low energy threshold of an order of sub $\mathrm{eV}$ is required for light WIMP searches. This is 3-4 orders of magnitude smaller than the level around $1-3 \mathrm{keV}$ for most heavy WIMP detectors [47, 49]. Germanium semiconductor detectors are widely used to study low energy neutrinos and WIMPs. The ionization energy is $0.67 \mathrm{eV}$. Thus it can be used in principle for energetic light WIMPs. In practice, their 


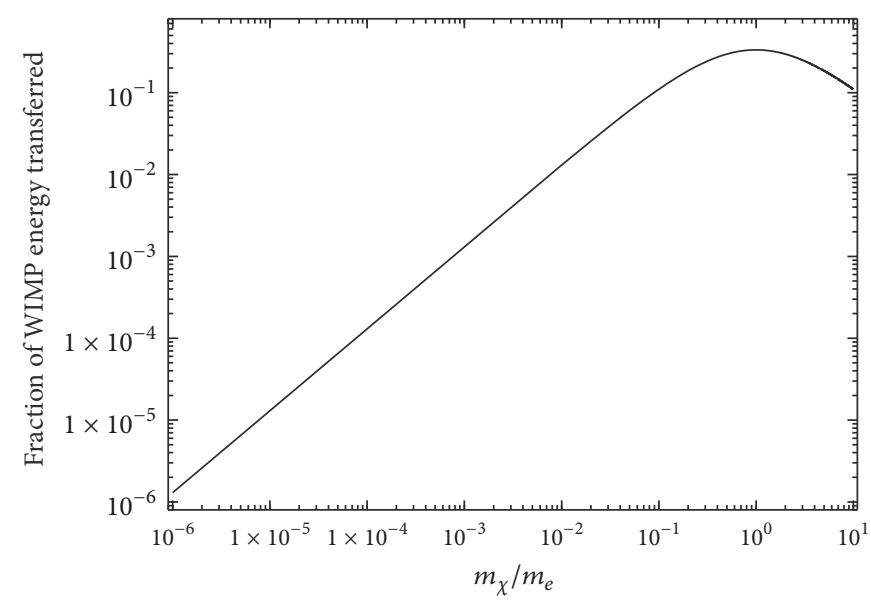

(a)

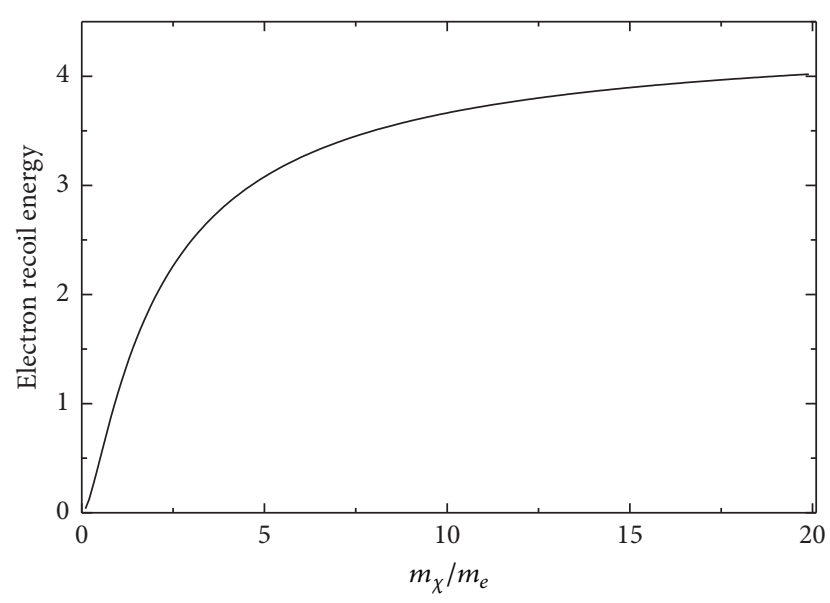

(b)

Figure 1: (a) The fraction of the energy of the WIMP that can be transferred to the electron as a function of $x=m_{\chi} / m_{e}$. (b) The electron recoil energy $T$ as a function of $x=m_{\chi} / m_{e}$. As $x$ increases it tends to $4.3 \mathrm{eV}$ for $y_{\mathrm{esc}}=2.84$.

threshold of around $200 \mathrm{eV}$ [50] or more is still far above the light WIMP signals. Bolometers are, in principle, low energy threshold and high energy resolution detectors, but the energy threshold of practical $10 \mathrm{~kg}$-scale bolometers is orders of magnitude higher than the light WIMP signal. Thus light WIMP detectors are necessarily different types from the present heavy WIMP detectors.

It is indeed a challenge to develop light WIMP detectors with low-threshold energy of the order of eV. Since the event rate is as large as $210^{3} / \mathrm{t} y$, one can use a small volume detector of the order of $10 \mathrm{kgr}$ at low temperature. In general, electric noises are random in time. Then, coincidence measurements of two signals are quite effective in reducing electric noise signals in case that one light WIMP produces 2 or more signals. One possible detector would be an ionization- scintillation detector, where one light WIMP interaction produces one ionized ion and one electron. In case that the ionized ion traps an electron nearby and emits a scintillation photon, one may measure the primary electron in coincidence with the scintillation photon. Nuclear emulsion may be of potential interest for low energy electrons. We briefly discuss possible new detection methods in Section 7.

\section{The Differential WIMP-Electron Rate}

The evaluation of the rate proceeds as in the case of the standard WIMP-nucleon scattering, but we will give the essential ingredients here to establish notation. We will begin by examining the case of a free electron.

4.1. Free Electrons. The differential cross section for WIMPelectron scattering in an atom can be cast in a form containing the electron recoil energy:

$$
T=\frac{q^{2}}{2 m_{e}}=\frac{(2 \mu r v \xi)}{2 m_{e}} .
$$

Thus the differential cross section takes the form

$$
\frac{d \sigma}{d T}=\sigma_{e} \frac{1}{2 v^{2}} \frac{m_{e}}{\mu_{r}^{2}},
$$

or

$$
\begin{gathered}
\frac{d \sigma}{d T}=\frac{1}{2 v^{2}} \sigma_{0} \frac{1}{m_{e}}, \\
\sigma_{0}=\sigma_{1} \frac{m_{e}^{2}}{m_{p}^{2}} .
\end{gathered}
$$

We find from (8) that the average energy of the electron is given by

$$
\begin{aligned}
&\langle T\rangle=\frac{2}{3(1+x)^{2}}\left\langle\beta^{2}\right\rangle m_{e} c^{2}, \\
&\left\langle\beta^{2}\right\rangle=\left\langle\left(\frac{v}{c}\right)^{2}\right\rangle=0.8 \times 10^{-6} .
\end{aligned}
$$

From (8) we also find that the fraction of the energy of the WIMP transferred to the electron, when taking $\left\langle\xi^{2}\right\rangle=1 / 3$ for scattering at forward angles, is

$$
\begin{aligned}
\frac{T}{T_{\chi}} & =\frac{4}{3} \frac{x}{(1+x)^{2}}, \quad x=\frac{m_{e}}{m_{\chi}}, \\
T_{\max } & =\frac{2 \mu_{r}^{2} v_{\mathrm{esc}}^{2}}{m_{e}} .
\end{aligned}
$$

This situation is exhibited in Figure 1. We thus see that this fraction attains a maximum when $x=1$, that is, when the two masses are equal. Away from this value it becomes smaller. The effect is more crucial for very light WIMPs, since their average energy is much smaller. Thus for $\mathrm{MeV}$ WIMPs the average energy transfer is in the $\mathrm{eV}$ region, which is reminiscent of the standard WIMPs where GeV mass leads 
to an energy transfer in the $\mathrm{keV}$ region. The maximum energy transfer corresponds to the escape velocity which is $v_{\text {esc }} \approx$ $2 \sqrt{\left\langle v^{2}\right\rangle}$, which leads to a value four times higher. The exact expression of the maximum electron energy will be given below.

From (8) we find that

$$
\begin{aligned}
v & =\sqrt{\frac{m_{e} T}{2 \mu_{r}^{2} \xi^{2}}} \longrightarrow \\
v & \geq \sqrt{\frac{m_{e} T}{2 \mu_{r}^{2}}} \longrightarrow \\
v_{\min } & =\sqrt{\frac{m_{e} T}{2 \mu_{r}^{2}}} .
\end{aligned}
$$

In other words the minimum velocity consistent with the energy transfer $T$ and the WIMP mass is constrained as above. The maximum velocity allowed is determined by the velocity distribution and it will be indicated by $v_{\text {esc }}$. From this we can obtain the differential rate per electron in a given velocity volume $v^{2} d v d \Omega$ as follows:

$$
d R=\frac{\rho_{\chi}}{m_{\chi}} v \sigma_{0} \frac{1}{2} \frac{1}{m_{e}} d T f(\boldsymbol{v}) d v d \Omega,
$$

where $f(\boldsymbol{v})$ is the velocity distribution of WIMPs in the laboratory frame. Integrating over the allowed velocity distributions we obtain

$$
\begin{aligned}
d R=\frac{\rho_{\chi}}{m_{\chi}} \sigma_{0} \frac{1}{2} \frac{1}{m_{e}} d T \eta\left(v_{\min }\right), & \\
\eta\left(v_{\min }\right) & =\int_{v_{\text {min }}}^{v_{\text {esc }}} f(\boldsymbol{v}) v d v d \Omega .
\end{aligned}
$$

$\eta\left(v_{\text {min }}\right)$ is a crucial parameter. Before proceeding further we find it convenient to express the velocities in units of the Sun's velocity. We should also take note of the fact the velocity distribution is given with respect to the center of the galaxy. For a M-B distribution this takes the form

$$
\frac{1}{\pi \sqrt{\pi}} e^{-y^{\prime 2}}, \quad y^{\prime}=\frac{v^{\prime}}{v_{0}}, v_{0}=220 \mathrm{~km} / \mathrm{s} .
$$

We must transform it to the local coordinate system:

$$
\mathbf{y}^{\prime} \longrightarrow \mathbf{y}+\widehat{v}_{s}+\delta\left(\sin \alpha \widehat{x}-\cos \alpha \cos \gamma \widehat{y}+\cos \alpha \sin \gamma \widehat{v}_{s}\right), \quad \delta=\frac{v_{E}}{v_{0}}
$$

with $\gamma \approx \pi / 6, \widehat{v}_{s}$ a unit vector in the Sun's direction of motion, $\hat{x}$ a unit vector radially out of the galaxy in our position, and $\widehat{y}=\widehat{v}_{s} \times \widehat{x}$. The last term in parenthesis in (17) corresponds to the motion of the Earth around the Sun with $v_{E} \approx 28 \mathrm{~km} / \mathrm{s}$ being the modulus of the Earth's velocity around the Sun and $\alpha$ the phase of the Earth $(\alpha=0$ around June 3rd). The above formula assumes that the motion of both the Sun around the Galaxy and of the Earth around the Sun are uniformly circular. Since $\delta$ is small, we expand the distribution in powers of $\delta$ and we keep terms up to linearity in $\delta$. Then (15) can be cast in the form

$$
\begin{aligned}
d R= & \left(\frac{\rho_{\chi}}{m_{\chi}} v_{0}\right) \sigma_{0} \frac{m_{t} Z_{\mathrm{eff}}}{A m_{p}} 1.9 \\
& \times 10^{6} \frac{1}{2} \frac{1}{m_{e}} d T\left(\Psi_{0}\left(y_{\min }\right)+\Psi_{1}\left(y_{\min }\right) \cos \alpha\right),
\end{aligned}
$$

where, in the above equation, the first term in parenthesis represents the average flux of WIMPs, the second provides the scale of the elementary cross section (in the present model the elementary cross section contains an additional mass dependence), the third term gives the number of electrons available for the scattering in a target of mass $m_{t}$ containing atoms with mass number $A$ and active electrons $Z_{\text {eff }}$, and the fourth is essentially the inverse of the square of the Sun's velocity in units of $c$ (its origin has its root in (10)). Moreover, $\Psi_{0}\left(y_{\min }\right)$ and $\Psi_{1}\left(y_{\min }\right)$ are functionals of $y_{\min }$ and $y_{\text {esc }}$, where

$$
\begin{aligned}
& y_{\min }=\frac{v_{\min }}{v_{0}}=\frac{1}{v_{0}} \sqrt{\frac{m_{e} T}{2 \mu_{r}^{2}}}, \\
& y_{\text {esc }}=\frac{v_{\text {esc }}}{v_{0}} .
\end{aligned}
$$

In the above expression the Heaviside function $H$ guarantees that the required kinematical condition is satisfied. One can factor the constants out in the above equation to get

$$
\begin{aligned}
& \frac{d R}{d(T / 1 \mathrm{eV})} \\
& =\Lambda\left(\Sigma_{0}\left(\frac{m_{\chi}}{m_{e}}, \frac{T}{1 \mathrm{eV}}\right)+\Sigma_{1}\left(\frac{m_{\chi}}{m_{e}}, \frac{T}{1 \mathrm{eV}}\right) \cos \alpha\right),
\end{aligned}
$$

where

$$
\begin{aligned}
\Sigma_{i}(t, s) & =\frac{1}{t} \Psi_{i}\left(1.23\left(1+\frac{1}{t}\right) \sqrt{s}\right), \quad i=0,1, \\
\Lambda & =1.4 \frac{\rho_{\chi}}{m_{e}} v_{0} \sigma_{0} \frac{m_{t} Z_{\mathrm{eff}}}{A m_{p}} .
\end{aligned}
$$

The meaning of $Z_{\text {eff }}$ will become clear after we consider the fact that the electrons are not free but bound in the atom. Thus they are not all available for scattering; that is, $Z_{\text {eff }}<Z$. We will now estimate $\Lambda$ considering the following input: (a) the 


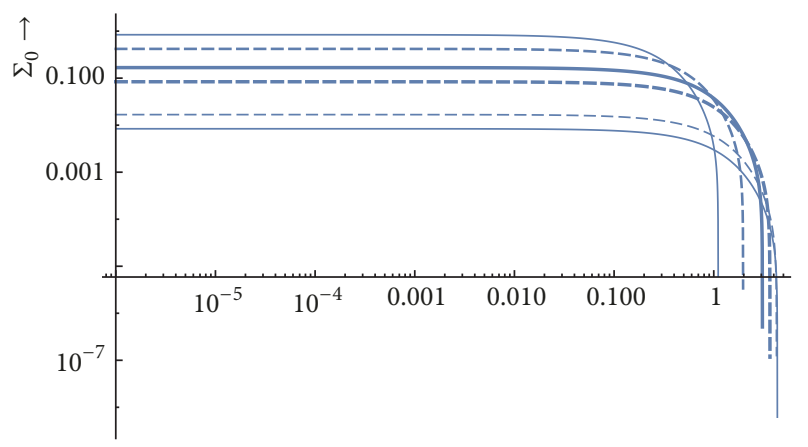

Figure 2: The shape of the differential cross section as a function of the electron energy $T$ in $\mathrm{eV}$ for the WIMP masses $(1,2,5,10,50,100) m_{e}$. The electrons are assumed to be free. The WIMP masses specifying the curves are increasing downwards. The spectrum does not exhibit any special structure.

elementary cross section $\sigma_{0}=5.0 \times 10^{-9} \mathrm{pb}=5.0 \times 10^{-45} \mathrm{~cm}^{2}$; (b) the total cross section, in units of $\sigma_{0}$, for example, $\sigma_{\mathrm{av}}=$ 0.2 for a WIMP mass about the electron mass (see below); (c) the particle density of WIMPs in our vicinity: $n=0.3 \times$ $10^{3}\left(\mathrm{MeV} / \mathrm{cm}^{3}\right) / 0.511 \mathrm{MeV} \approx 600 \mathrm{~cm}^{-3}$ (we use the electron mass in this estimate; the correct mass dependence has been included in evaluating $\sigma_{\text {av }}$ ); this value leads to a flux: $n \times$ $220 \mathrm{~km} / \mathrm{s}=1.3 \times 10^{10} \mathrm{~cm}^{-2} \mathrm{~s}^{-1}$; (d) The number of electrons in a Kg of Xe: $\left(1 /\left(131 \times 1.67 \times 10^{-27}\right)\right) Z_{\text {eff }}=4.6 \times 10^{24} Z_{\text {eff }}$. Taking $Z_{\text {eff }}=54$, that is, all electrons in Xe participating, we expect about $\approx 0.3$ events per $\mathrm{kg} \cdot \mathrm{y}$. Encouraged by this estimate, even though it has been obtained with a much smaller elementary cross section than previous estimates [32], we are going to proceed in evaluating the expected spectrum of the recoiling electrons.

The shape of the differential cross section $\Sigma_{0}(t, s)$ as a function of the electron energy $T$ in $\mathrm{eV}$ for various WIMP masses is exhibited in Figure 2. The electrons are assumed to be free. Obviously, this does not exhibit any special structure.

The various atomic physics approximations previously considered involve relatively high electron energies, as, for example, in the recent works $[17,18]$, which are not important in our case. The obtained rate, however, can be modified substantially at low energies by including the correction of the outgoing electron wave due to the Coulomb field. In beta decay this is done via the simple Fermi function [51]:

$$
\begin{aligned}
F & (k, Z, \eta, \gamma) \\
& =(k R)^{2 \gamma-2} e^{\pi \nu}\left|\frac{\Gamma(\gamma+i \eta)}{\Gamma(2 \gamma)}\right|^{2}|M(\gamma+i \eta, 2 \gamma, 2 i k r)|^{2},
\end{aligned}
$$

where

$$
\begin{aligned}
& \gamma=\sqrt{1-\alpha^{2} Z^{2}}, \\
& \eta=Z \alpha \frac{T+m_{e} c^{2}}{\sqrt{T^{2}+2 m_{e} c^{2} T}}
\end{aligned}
$$

and $M(\gamma+i \eta, 2 \gamma, 2 i k r)$ is the Coulomb function represented by a confluent hypergeometric function.

After some approximations (see, e.g., Landau's book [52]) one finds the Fermi function

$$
\begin{aligned}
& F(T, Z, n, \eta, \gamma) \\
& \quad \approx\left(\sqrt{T^{2}+2 m_{e} T} \bar{r}(n, Z, \gamma)\right)^{2 \gamma-2} f_{c}^{2}(\gamma, n) \frac{2 \pi \eta}{1-e^{-2 \pi \eta}} .
\end{aligned}
$$

This function, which depends on the electron energy as well as the atomic parameters $n$ and $Z$, may lead to an enhancement for low energy electrons. Integrating the differential rate given by (20) over the electron spectrum we obtain the total rate:

$$
R=\Lambda\left(\sigma_{\mathrm{av}}+\sigma_{\mathrm{td}} \cos \alpha\right)
$$

where $\sigma_{\mathrm{av}}$ and $\sigma_{\mathrm{td}} \cos \alpha$ are the average and time dependent (modulated) cross sections, respectively, in units of $\sigma_{0}$, that is, of the elementary cross section. The obtained quantity $\sigma_{\mathrm{av}}$ is shown in Figure 3 for free electrons both without the Fermi function as well as with the Fermi function for two values $n$ and $Z, n$ assumed to be sort of average. We see that the effect on the total cross section is large. Thus we find that the cross sections (in units of $\sigma_{0}$ ), for a WIMP with the mass of the electron we get values $0.2,0.8$, and 2.2 for cases (a), (b), and (c) respectively (see Figure 3), while for $n=2$ and $Z=50$ we find 0.3 . In our estimates we will adopt an average enhancement factor of 8 due to the Fermi function taking into account the Fermi function the above estimate becomes 2.5 events/(kg.y).

\section{Effects of Binding of Electrons}

The binding of the electrons comes in two ways. The first is the most obvious. A portion of the energy of the WIMP will not go to recoil, but it will be spent to release the bound electron. The second comes from the fact that the initial electron is not at rest but it has a momentum distribution, which is the Fourier transform of its wave function in coordinate space.

Since the propagator involves the $Z$ - exchange with a large mass, the momentum transfer can be neglected. So the cross section for WIMP-electron scattering is proportional to

$$
J=\frac{1}{(2 \pi)^{2}} \int d^{3} \mathbf{p}\left|\widetilde{\phi}_{n \ell}(\mathbf{p})\right|^{2} d^{3} \mathbf{q} d^{3} \mathbf{p}_{\chi}^{\prime} \delta\left(\mathbf{p}+\mathbf{p}_{\chi}-\mathbf{p}_{\chi}^{\prime}-\mathbf{q}\right) \delta\left(\frac{\mathbf{p}_{\chi}^{2}}{2 m_{\chi}}-\frac{\mathbf{p}_{\chi}^{\prime 2}}{2 m_{\chi}}-\frac{\mathbf{q}^{2}}{2 m_{e}^{2}}-b\right)
$$

where $\widetilde{\phi}_{n \ell}(\mathbf{p})$ is the electron wf in momentum space. A plane wave outgoing electron has been assumed. Deviations from plane wave will be accounted for in the usual way, that is, by the Fermi function as described in the previous section. After 


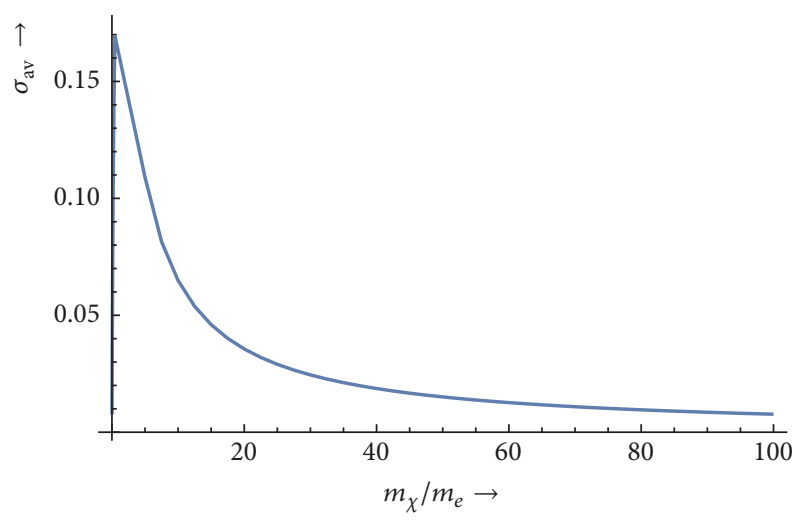

(a)

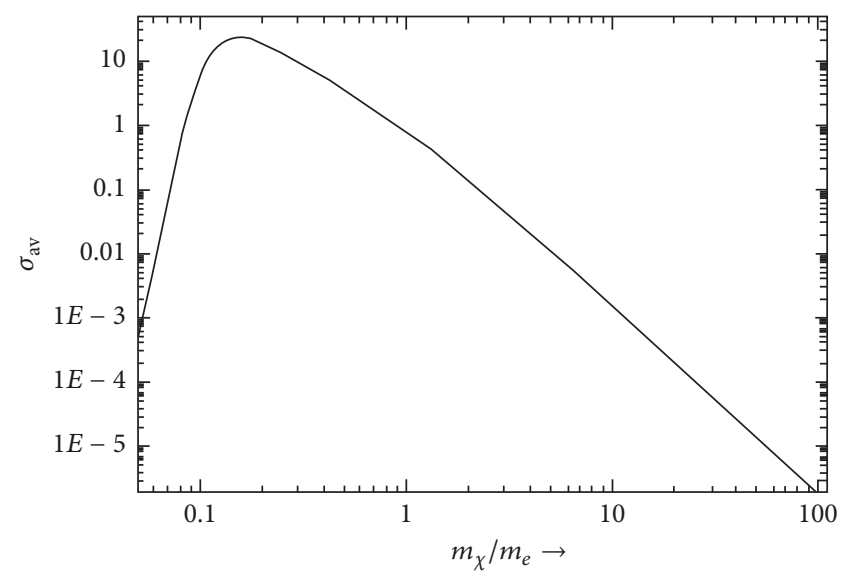

(b)

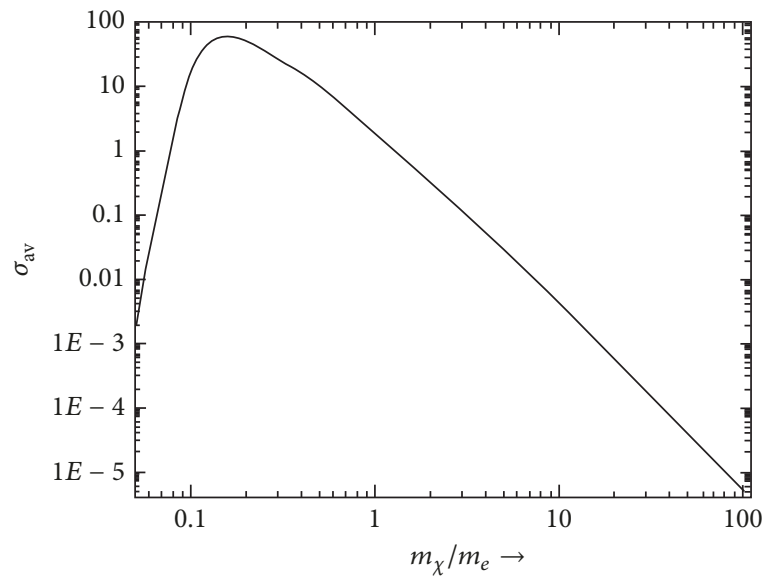

(c)

FIGURE 3: The total average WIMP-electron cross section in units $\sigma_{0}$ as a function of the WIMP mass in units of the electron mass. In panel (a) the Fermi function was neglected, while in panels (b) and (c) the Fermi function $F$ for $n=3$ and $Z=50$ and $n=4$ and $Z=60$, respectively, has been employed.

the integration over the momentum $\mathbf{p}$ via the $\delta$ function we obtain

$$
J=\frac{1}{(2 \pi)^{2}} \int\left|\widetilde{\phi}_{n \ell}\left(\mathbf{p}_{\chi}^{\prime}-\mathbf{p}_{\chi}+\mathbf{q}\right)\right|^{2} d^{3} \mathbf{p}_{\chi}^{\prime} d^{3} \mathbf{q} \delta\left(\frac{\mathbf{p}_{\chi}^{2}}{2 m_{\chi}}-\frac{\mathbf{p}_{\chi}^{\prime 2}}{2 m_{\chi}}-\frac{\mathbf{q}^{2}}{2 m_{e}^{2}}-b\right)
$$

The integration over the magnitude of $\mathbf{p}_{\chi}^{\prime}$ can be done using the energy conserving $\delta$ function and we obtain

$$
\begin{array}{r}
J=\frac{1}{(2 \pi)^{2}} \int d^{3} \mathbf{q} Q^{2} d \Omega_{\mathbf{Q}}\left|\widetilde{\phi}_{n \ell}\left(\mathbf{Q}-\mathbf{p}_{\chi}+\mathbf{q}\right)\right|^{2} \frac{m_{\chi}}{Q}, \\
\mathbf{Q}=\widehat{e} \sqrt{\left(m_{\chi} v\right)^{2}-q^{2} x-2 m_{\chi} b}
\end{array}
$$

with $x=m_{\chi} / m_{e}$ and $\hat{e}$ a unit vector in the direction of $\mathbf{p}_{\chi}^{\prime}$. We thus find the important constraint

$$
v>v_{\min }, \quad v_{\min }=\sqrt{\frac{2}{m_{\chi}}(T+b) .}
$$

This already sets a limit on the range of the variables $b$ and $T$ of interest to experiments. 


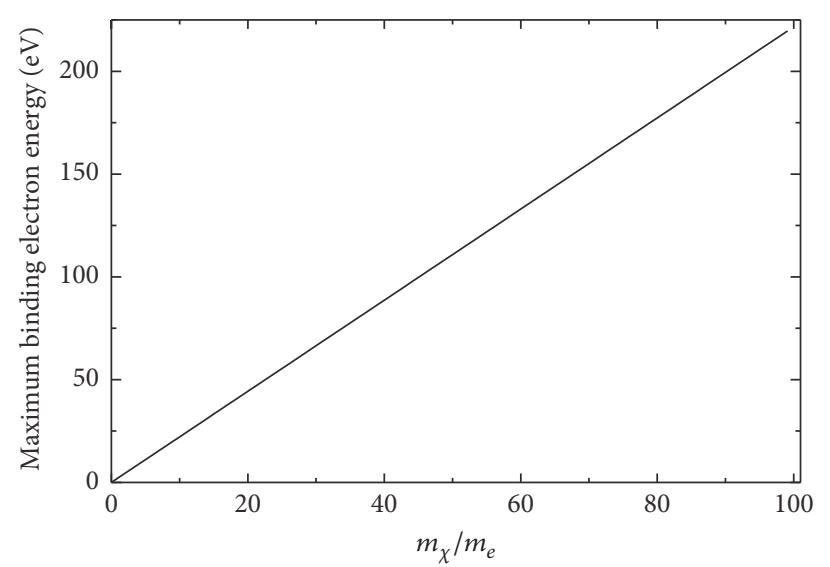

FIGURE 4: The maximum binding electron energy $b_{\max }$ in $\mathrm{eV}$ as a function of $m_{\chi} / m_{e}$ accessible to WIMP-electron scattering.

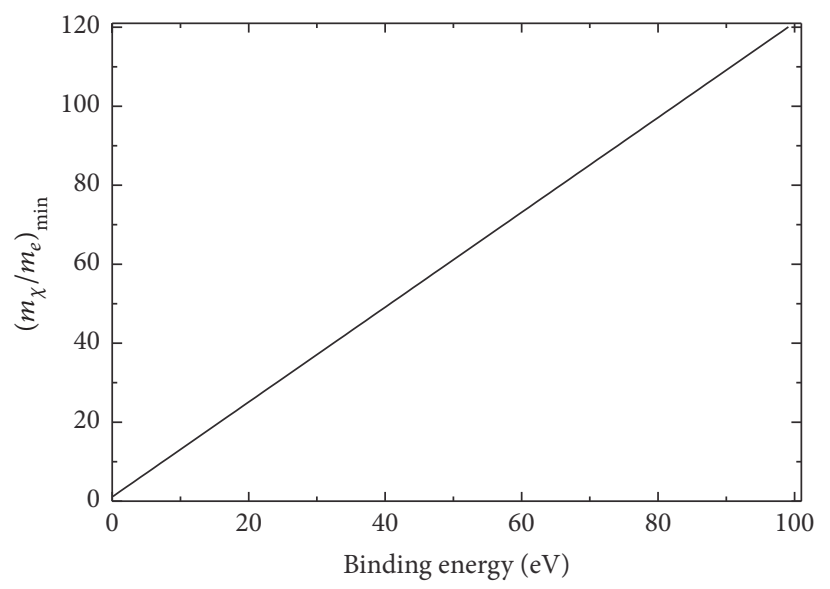

(a)

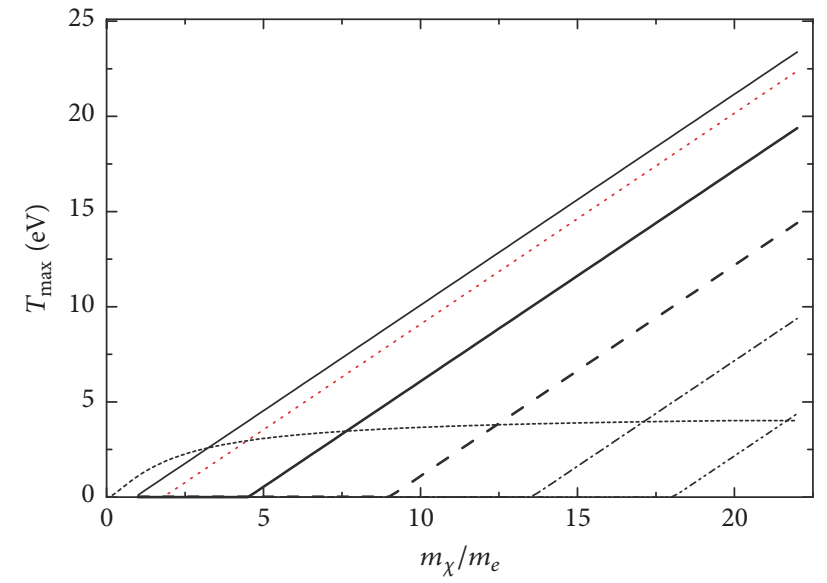

(b)

FIGURE 5: The minimum $m_{\chi} / m_{e}$ required to eject an electron with binding energy $b$ in $\mathrm{eV}$ (a). The maximum outgoing electron energy $T_{\max }$ as a function of $x=m_{\chi} / m_{e}$ for various binding energies $b$ (b). In this panel the graphs correspond to $b=1,2,5,10,15$, and $20 \mathrm{eV}$ from left to right. For convenience we also show again the curve which corresponds to a free electron (long dashed). It yields the same maximum electron energy for $b=20 \mathrm{eV}$ and $x=22$.

5.1. The Allowed Range of Binding Energy $(b)$ and Recoil Energy $(T)$. The range of these quantities is not affected by the specific form of the bound electron wave function. In particular regarding $b$ the above conditions imply

$$
\begin{aligned}
(T)_{\max } & =\frac{1}{2} m_{\chi} v_{\mathrm{esc}}^{2}-b, \\
b_{\max } & =\frac{1}{2} m_{\chi} v_{\mathrm{esc}}^{2},
\end{aligned}
$$

where $b_{\max }$ is associated with zero maximum recoil energy and is exhibited as a function of WIMP mass in Figure 4. What seriously affects the detection of light WIMPs in the presence of large binding energies is the minimum WIMP velocity required to eject an electron. One finds that in order to surpass the barrier of a given binding energy $b$ the WIMP must have a minimum velocity at most $v_{\text {esc }}$ and a high mass, even if the electron energy is zero. The minimum $x=m_{\chi} / m_{e}$ required for this purpose is exhibited in Figure 5(a). The actual value of $x=m_{\chi} / m_{e}$ must, of course, be larger to get a reasonable rate.

Finally we present in Figure 5(b) the maximum possible energy for outgoing electrons as a function of the WIMP mass for various binding energies. We see that the maximum energy of the electron increases due to the binding provided that the binding energy is small. Thus the threshold energy needs not be extremely low, provided that for $x \leq 20$ there exist enough electrons in the atom with low enough binding energies. For heavier WIMPs the binding energy of the electron plays a minor role and the electron recoil energy increases linearly with the WIMP mass.

5.2. The Effect of the Bound Electron Wave Function. To proceed further we must evaluate the integral in (28). Firstly, we must select a suitable coordinate system, for example, one with the $z$-axis along the initial WIMP velocity, the $x$-axis in the direction of the outgoing WIMP, and $y$-axis perpendicular to the plane of the other two. Actually, there 
is no hope for obtaining an analytic expression even for the angular integrals without further approximations. So we will assume hydrogenic wave functions. This approximation is expected to be reasonable for electrons which are not tightly bound, which are of interest to us in the present work. We concentrate on the case of $s$ states and find that the cross section is given by

$$
d \sigma=\frac{1}{v}|\mathscr{M}|^{2} d J
$$

where $d J$ is a complicated functional given by

$$
\begin{aligned}
& d J=\frac{q}{p_{0}(n, Z)} q d q \psi_{2}\left(q, v, b, p_{0}(n, Z)\right), \\
& \psi_{2}\left(q, v, b, p_{0}(n, Z)\right)=\int_{-1}^{1} d \xi \psi_{1}\left(\xi, q, v, b, p_{0}(n, Z)\right),
\end{aligned}
$$

where

$$
\begin{aligned}
\psi_{1} & \left(\xi, q, v, b, p_{0}(n, Z)\right) \\
& =\frac{m_{\chi}}{\left(q \xi-m_{\chi} v\right)} \int_{\eta_{+}}^{\eta_{-}} z d z\left|\widetilde{\phi}_{n \ell}(z)\right|^{2}
\end{aligned}
$$

and $p_{0}(n, Z)=(\alpha Z / n) m_{e}$, the scale of momentum of the bound electron wave function. $\mathscr{M}$ is the invariant amplitude for the process which has the form

$$
|\mathscr{M}|^{2}=\sigma_{e} \frac{\pi}{\mu_{r}^{2}}=\sigma_{0} \frac{\pi}{m_{e}^{2}} .
$$

Now, since

$$
d \sigma=\sigma_{0} \frac{1}{v} \frac{\pi}{m_{e}^{2}} d J
$$

one can cast (35) in a form similar to the expression for free electrons, namely,

$$
d \sigma=\sigma_{0} \frac{1}{2 v^{2}} \frac{1}{m_{e}} d T
$$

or

$$
d \sigma=\sigma_{0} \frac{1}{v^{2}} \frac{1}{m_{e}} d T \widetilde{\Lambda}\left(T, v, b, p_{0}(n, Z)\right),
$$

where

$$
\begin{aligned}
& \widetilde{\Lambda}\left(T, v, b, p_{0}(n, Z)\right) \\
& \quad=2 \pi v \frac{\sqrt{2 m_{e} T}}{p_{0}(n, Z)} \psi_{2}\left(\sqrt{2 m_{e} T}, v, b, p_{0}(n, Z)\right) .
\end{aligned}
$$

In order to get the differential rate one must fold the above expression with the velocity distribution $f(\boldsymbol{v})$ in a similar fashion as in (15), that is,

$$
\begin{aligned}
& \frac{d R}{d T}=\frac{\rho_{\chi}}{m_{\chi}} \frac{1}{2} \sigma_{0} \frac{1}{m_{e}}\left[\int_{\sqrt{2(b+T) / m_{\chi}}}^{v_{\text {esc }}} \tilde{\Lambda}\left(T, v, b, p_{0}(n, Z)\right)\right. \\
& \cdot f(\boldsymbol{v}) v d v d \widehat{\boldsymbol{v}}] .
\end{aligned}
$$

Note the appearance of the quantities $b$ and $\widetilde{\Lambda}$ as a result of the electron binding. The behavior of the function $\widetilde{\Lambda}$ as a function of the velocity and its numerical value significantly affects the obtained rates. In order to obtain $\widetilde{\Lambda}$ exactly one must evaluate the remaining integrals numerically for each electron orbit separately. After that, in order to obtain the total rate, one must sum up over all available electrons involved in any orbit of a given atom, which is not trivial. This is currently under study, but here we will report results obtained in an approximate scheme valid for relatively low mass WIMPs, which is adequate for our purposes.

5.3. A Convenient Approximation for Light WIMPs. We have seen that the momentum of the outgoing electron is small compared to $p_{0}(n, Z)$. Let us assume that

$$
\frac{Q m_{\chi} v}{p_{0}^{2}(n, Z)}<1 \text {. }
$$

This means that

$$
x^{2} v \sqrt{v-v_{1}} \leq \alpha^{2}, \quad v_{1}=\sqrt{\frac{2(b+T)}{x m_{e}}} \leq v_{\mathrm{esc}} .
$$

This quantity is small as long as

$$
x<\frac{(\alpha Z)}{v_{\mathrm{esc}}}=1.2 Z \approx 50 \text { for a large atom. }
$$

For such values of $x$ we can expand the integral $\int_{\eta_{+}}^{\eta_{-}} z d z\left|\widetilde{\phi}_{n \ell}(z)\right|^{2}$ up to second order in the small parameter. The result, for example, for $1 \mathrm{~s}$ hydrogenic wave functions, is

$$
J_{1}=\frac{16 Q\left(m_{\chi} v-q \xi\right)}{\pi^{2} p_{0}^{2}(n, Z)} .
$$

Integrating over the angles of the outgoing electron we obtain

$$
d J=q^{2} d q \frac{64 Q^{2} m_{\chi} m_{\chi} v}{\pi p_{0}^{5}(n, Z)} .
$$

Proceeding as above we obtain

$$
\widetilde{\Lambda}=\widetilde{\Lambda}_{0}(Z) \sqrt{\frac{2 T}{m_{e}}} \frac{x^{4}}{(\alpha Z)^{5}} v^{2}\left(v^{2}-v_{1}^{2}\right)
$$

or

$$
\begin{aligned}
& \widetilde{\Lambda}=\widetilde{\Lambda}_{0}(Z) x^{4} \sqrt{T} y^{2}\left(y^{2}-\frac{6.59(b+T)}{x}\right), \\
& \widetilde{\Lambda}_{0}(1)=3.5 \times 10^{-3},
\end{aligned}
$$

where $b$ and $T$ are in $\mathrm{eV}$ and $y$ is the WIMP velocity in units of the Sun's velocity. A similar expression with a slightly different constant $\widetilde{\Lambda}_{0}(Z)$ is expected to hold for other electron orbitals. This function must be multiplied with the velocity distribution before proceeding with the needed integrations to obtain the rate. 


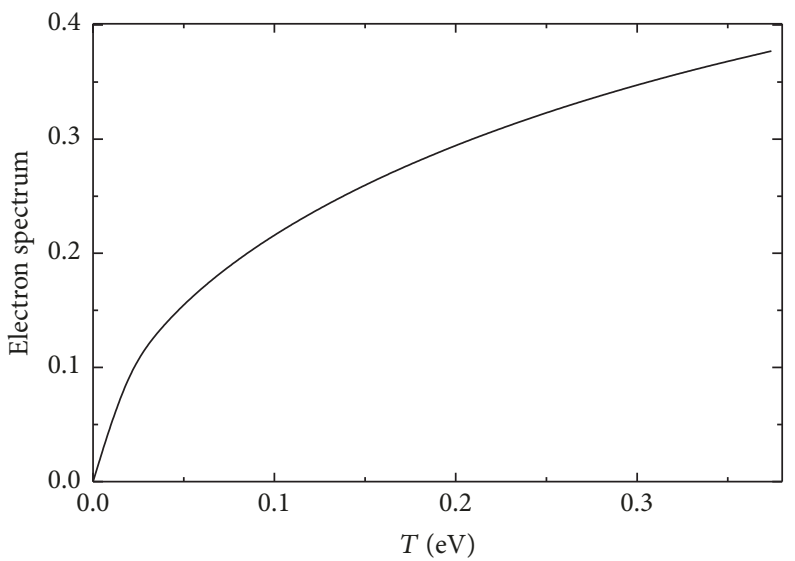

FIGURE 6: The electron spectrum for bound electrons corresponding to $x=1$. Only electrons with $b<1 \mathrm{eV}$ can be ejected.

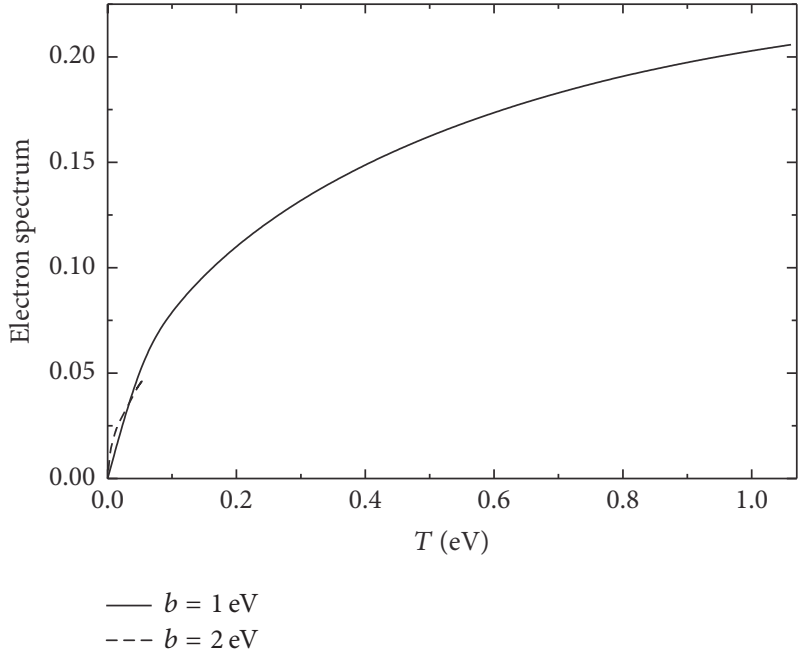

(a)

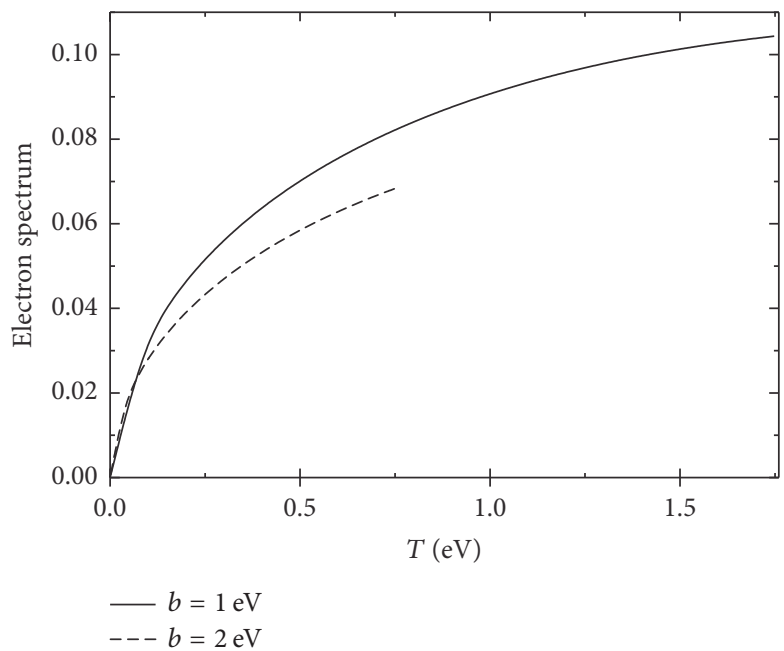

(b)

Figure 7: The same as in Figure 6, but for $x=10$ in panel (a) and $x=20$ in panel (b). In both cases only electrons with $b<3 \mathrm{eV}$ can be ejected. Notice, however, that the spectrum is suppressed for $b=2$ (dashed line) compared to the corresponding one for $b=1$ (solid line).

\section{Some Results for Bound Electrons}

We will limit ourselves to $b \geq 1 \mathrm{eV}$ and $x \leq 100$ After integrating with the velocity distribution we obtain the electron spectra shown in Figures 6-8. After integrating over the energy spectrum we obtain the cross section $\sigma_{\mathrm{av}}$ in units of $\sigma_{0}$ shown in Figure 9 as a function of the WIMP mass for various binding energies. It is perhaps better to show $\sigma_{\mathrm{av}}$ as a function of the binding energy. For $x=10$ only $b \leq 1$ are available. For $b=1$ we find $\sigma_{\mathrm{av}}=0.1$ in units of $\sigma_{0}$. For larger $x, \sigma_{\mathrm{av}}$ is exhibited in Figure 10 .

We thus see that using hydrogenic wave functions we find that, for $m_{\chi}$, greater $10 m_{e}$, electrons with $b<15 \mathrm{eV}$ become available. The number of electrons with relatively small binding energy for some targets of interest is shown in Table 1. Anyway there seems to be a wide range of the parameters which leads to $Z_{\text {eff }}=5$ for a suitable atom with large $Z$. Thus for $Z_{\text {eff }}=5$ one can conservatively set $\sigma_{\text {av }}$ to be $\approx 0.15$, which leads to about 0.6 event per kg-y compared to the 2.0 per kg-y we got above for lighter WIMPs without the electron binding. The Fermi function is incorporated into the results.

\section{Discussion}

We have seen that the use of electron detectors may be a good way for directly detecting light WIMPs in the $\mathrm{MeV}$ region. The electron density in our vicinity is very high, the elementary WIMP-electron cross section may be quite large, and the event rate may be further enhanced by the behavior of the Fermi function at low energies. This is fine for WIMPs heavier than 20 times the electron mass. For WIMPs less than about 20 times the mass of the electron, however, there appears to be a problem arising from the electron binding. A small fraction of electrons can be exploited, namely, those electrons with binding energies below the $10 \mathrm{eV}$ range. This is reminiscent of the difficulty encountered in the inelastic WIMP nucleus scattering, whereby only very low excited 


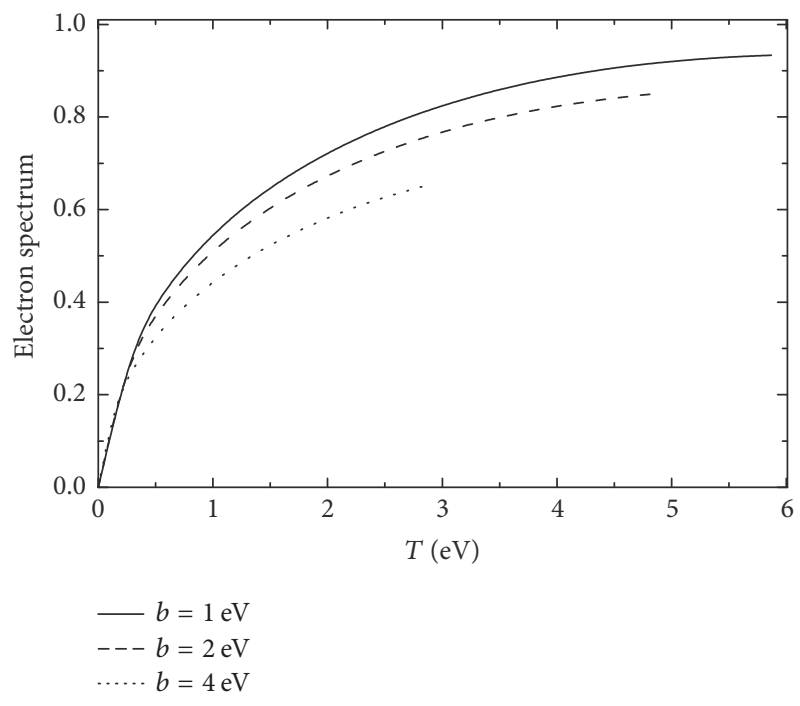

(a)

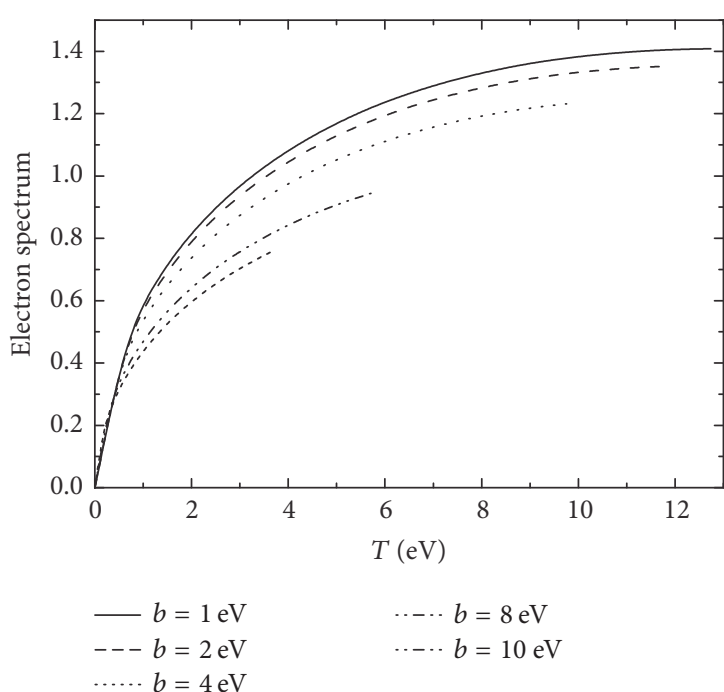

(b)

Figure 8: The same as in Figure 7, but for $x=50$ in panel (a) and $x=100$ in panel (b). In panel (a) the spectrum is shown for $b=1,2$ and $4 \mathrm{eV}$. In panel (b) the spectra for $b=1,2,4$ and $8 \mathrm{eV}$ are shown with $b$ increasing downwards.

TABLE 1: The number of electrons with binding energies less than $b_{\mathrm{upb}}$ for a given set of targets.

\begin{tabular}{|c|c|c|c|c|c|}
\hline Target & $b_{\mathrm{upb}}=5 \mathrm{eV}$ & $b_{\text {upb }}=10 \mathrm{eV}$ & $b_{\text {upb }}=15 \mathrm{eV}$ & $b_{\mathrm{upb}}=20 \mathrm{eV}$ & $b_{\mathrm{upb}}=30 \mathrm{eV}$ \\
\hline${ }_{9} \mathrm{~F}$ & - & - & - & 5 & 5 \\
\hline${ }_{11} \mathrm{Na}$ & 1 & 1 & 1 & 1 & 1 \\
\hline${ }_{32} \mathrm{Ge}$ & 4 & 4 & 4 & 4 & 14 \\
\hline${ }_{52} \mathrm{Te}$ & 4 & 4 & 6 & 6 & 6 \\
\hline${ }_{53} \mathrm{I}$ & 5 & 5 & 7 & 7 & 7 \\
\hline${ }_{54} \mathrm{Xe}$ & - & - & 6 & 6 & 8 \\
\hline${ }_{83} \mathrm{Bi}$ & 3 & 5 & 5 & 5 & 15 \\
\hline
\end{tabular}

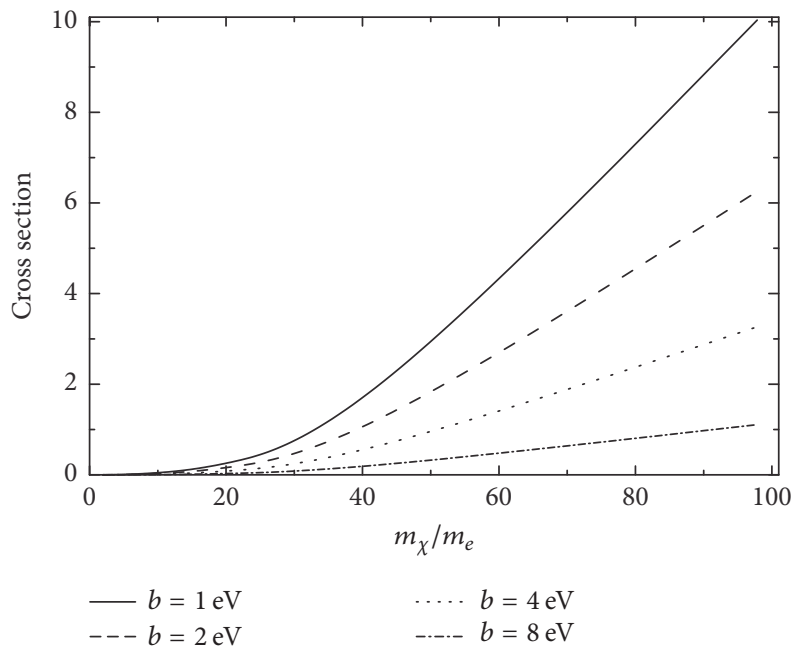

FIGURE 9: The cross section $\sigma_{\mathrm{av}}$ in units of $\sigma_{0}$ as a function of $x=m_{\chi} / m_{e}$ for binding energies $b=1,2,4$, and $8 \mathrm{eV}$ increasing downwards (the curve for $b=10$ is not visible).

states can be reached. We have seen, however, that, due to the behavior of the Fermi function in the case of very low energy electron recoils, the rates may increase up to factors of 8 . So event rates of about 0.8 to 2.4 events per kg-y are possible.

It has recently been suggested that it is possible to detect even very light WIMPs, much lighter than the electron, utilizing Fermi-degenerate materials like superconductors [53]. In this case the energy required is essentially the gap energy of about $1.5 k T_{c}$, which is in the meV region; that is, the electrons are essentially free. The authors are perhaps aware of the fact that the average energy for very light WIMPs is small and not all of it can be transferred to their system (the maximum fraction occurs if the mass of the WIMP is equal to $m_{e}$, i.e., for $x=1$ in Figure 1). With their detector these authors probably have a way to circumvent the fact that a small amount of energy will be deposited, if they manage to accumulate a large number of loosely bound electrons in their targets so that the obtained rates are sufficient. More recently it is claimed that even smaller energies in $\mathrm{meV}$ can be detected in the case of Liquid Helium [54]. The expected event rates and the total energy deposited in such essentially bolometer type detectors are currently being estimated more precisely and they will appear elsewhere.

It thus appears that light WIMPs in the $\mathrm{MeV}$ region can, in principle, be detected. The detection techniques and targets 


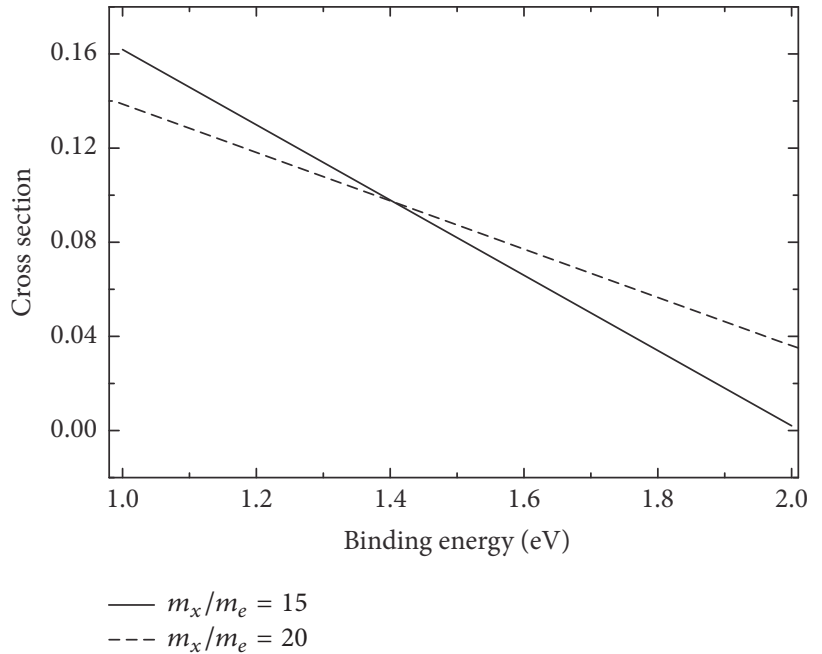

(a)

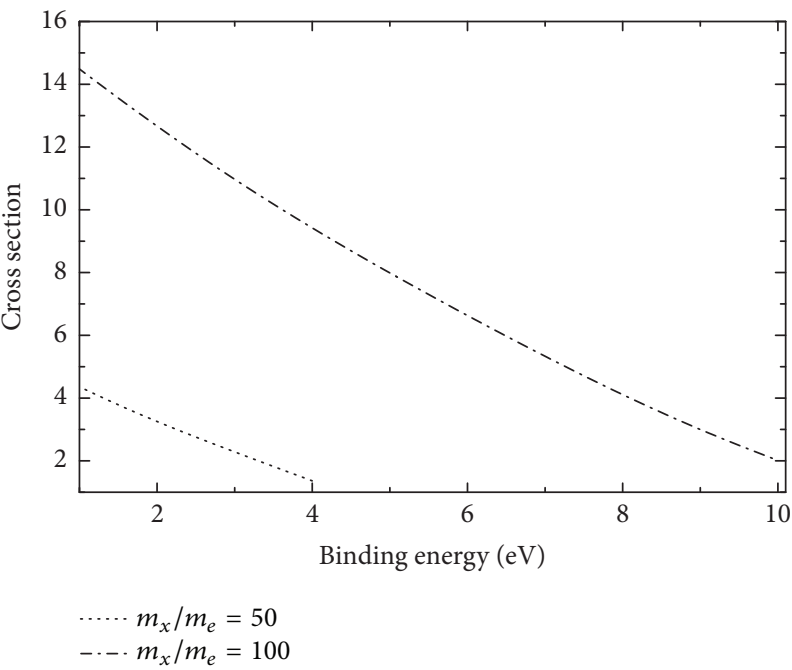

(b)

FIgURE 10: The cross section $\sigma_{\mathrm{av}}$ in units of $\sigma_{0}$ as a function of $b$ in eV for $x=m_{\chi} / m_{e}=15$ (solid line) and 20 (dashed line) (a) and for $x=m_{\chi} / m_{e}=50$ and 100 (b) (the lower curve corresponds to the smaller $x$ ).

employed, however, may have to be different than the ones employed in standard WIMP searches.

\section{Disclosure}

The permanent address of J. D. Vergados is University of Ioannina, 45110 Ioannina, Greece.

\section{Conflicts of Interest}

The authors declare that there are no conflicts of interest regarding the publication of this paper.

\section{Acknowledgments}

J. D. Vergados is happy to acknowledge support of this work by (i) the National Experts Council of China via a "Foreign Master" grant and (ii) IBS-R017-D1-2016-a00 in the Republic of Korea. A substantial part of this work performed while J. D. Vergados was on a visit to the University of Adelaide, supported by CoEPP and the Centre for the Subatomic Structure of Matter (CSSM). He is happy to thank Professors Yannis K. Semertzidis of KAIST, Anthony Thomas of Adelaide, and Edna Cheung of Nanjing University for their hospitality. Yeuk-Kwan E. Cheung acknowledges support from the Jiangsu Ministry of Science and Technology under Contract BK20131264 and the Priority Academic Program Development for Jiangsu Higher Education Institutions (PAPD).

\section{References}

[1] S. Hanary, "MAXIMA-1: a measurement of the cosmic microwave background anisotropy on angular scales of $10^{\prime}-5^{\circ}$," The Astrophysical Journal, vol. 545, no. 1, pp. L5-L10, 2000.
[2] J. H. P. Wu, A. Balbi, J. Borrill et al., "Tests for Gaussianity of the MAXIMA-1 Cosmic Microwave Background Map," Physical Review Letters, vol. 87, article 251303, 2001.

[3] M. G. Santos, A. Balbi, J. Borrill et al., "Estimate of the Cosmological Bispectrum from the MAXIMA-1 Cosmic Microwave Background Map," Physical Review Letters, vol. 88, article 241302, 2002.

[4] P. D. Mauskopf, P. A. Ade, and P. de Bernardis, "Measurement of a peak in the cosmic microwave background power spectrum from the North American test flight of boomerang," The Astrophysical Journal, vol. 536, no. 2, pp. L59-L62, 2002.

[5] S. Mosi, P. de Bernardis, and G. de Troia, "The BOOMERanG experiment and the curvature of the universe," Progress in Particle and Nuclear Physics, vol. 48, no. 1, pp. 243-261, 2002.

[6] N. W. Halverson, E. M. Leitch, and C. Pryke, "Degree Angular Scale Interferometer First Results: A Measurement of the Cosmic Microwave Background Angular Power Spectrum," The Astrophysical Journal, vol. 568, article 38, 2002.

[7] G. F. Smoot, C. L. Bennett, A. Kogut et al., "Structure in the COBE differential microwave radiometer first-year maps," Astrophysical Journal, vol. 396, article L1, 1992.

[8] A. H. Jaffe, P. A. Ade, A. Balbi et al., "Cosmology from MAXIMA-1, BOOMERANG, and COBE DMR Cosmic Microwave Background Observations," Physical Review Letters, vol. 86, article 3475, 2001.

[9] D. N. Spergel, L. Verde, and H. V. Peiris, "First Year Wilkinson Microwave Anisotropy Probe (WMAP) Observations: Determination of Cosmological Parameters," The Astrophysical Journal, vol. 148, article 175, 2003.

[10] D. N. Spergel, R. Bean, O. Doré et al., "Wilkinson Microwave Anisotropy Probe (WMAP) Three Year Results: Implications for Cosmology," The Astrophysical Journal Supplement Series, vol. 170, article 377, 2007.

[11] The Planck Collaboration, P. A. R. Ade, N. Aghanim, C. Armitage-Caplan et al., "Planck 2013 results. XVI. Cosmological parameters," Astronomy \& Astrophysics, 2013. 
[12] D. P. Bennett, C. Alcock, R. A. Allsman et al., "Experimental Limits on the Dark Matter Halo of the Galaxy from Gravitational Microlensing," PHysical Review Letters, vol. 74, article 2867, 1995.

[13] P. Ullio and M. Kamioknowski, "Velocity distributions and annual-modulation signatures of weakly-interacting massive particles," Journal of High Energy Physics, vol. 2001, no. 03, p. 049, 2001.

[14] J. Vergados and H. Ejiri, "The role of ionization electrons in direct neutralino detection," Physics Letters B, vol. 606, no. 3-4, pp. 313-322, 2005.

[15] H. Ejiri, C. C. Moustakidis, and J. D. Vergados, "Dark matter search by exclusive studies of X-rays following WIMPs nuclear interactions," Physics Letters B, vol. 639, no. 3-4, pp. 218-222, 2006.

[16] C. C. Moustakidis, J. D. Vergados, and H. Ejiri, "Direct dark matter search by observing electrons produced in neutralino-nucleus collisions," Nuclear Physics B, vol. 727, article 406, 2005.

[17] B. Roberts, V. Flambaum, and G. Gribakin, "Ionization of atoms by slow heavy particles, including dark matter," Physical Review Letters, vol. 116, article 023201, 2016.

[18] B. M. Roberts, V. A. DZUba, V. V. Flambaum et al., "Dark matter scattering on electrons: Accurate calculations of atomic excitations and implications for the DAMA signal," Physical Review D, vol. 93, article 115037, 2016.

[19] J. D. Lewin and P. F. Smith, "Review of mathematics, numerical factors, and corrections for dark matter experiments based on elastic nuclear recoil," Astroparticle Physics, vol. 6, article 87, 1996.

[20] V. Barger, M. Ishida, and W. Keung, "Total Width of $125 \mathrm{GeV}$ Higgs Boson," Physical Review Letters, vol. 108, no. 26, 2012.

[21] M. W. Goodman and E. Witten, "Detectability of certain dark-matter candidates," Physical Review D: Particles, Fields, Gravitation and Cosmology, vol. 31, no. 12, pp. 3059-3063, 1985.

[22] A. K. Drukier, K. Freese, and D. N. Spergel, "Detecting cold dark-matter candidates," Physical Review D: Particles, Fields, Gravitation and Cosmology, vol. 33, no. 12, pp. 3495-3508, 1986.

[23] J. R. Primack, D. Seckel, and B. Sadoulet, "Detection of Cosmic Dark Matter," Annual Review of Nuclear and Particle Science, vol. 38, article 751, 1988.

[24] A. Gabutti and K. Schmiemann, "Detection rate of weakly interacting massive particles," Physics Letters B, vol. 308, no. 3-4, pp. 411-417, 1993.

[25] R. Bernabei, "Research on dark matter," Riv. Nuovo Cimento, vol. 18, no. 5, pp. 1-68, 1995.

[26] D. Abriola, F. T. AvignoneIII, and R. L. Brodzinski, "Search for an annual modulation of dark-matter signals with a germanium spectrometer at the Sierra Grande Laboratory," Astroparticle Physics, vol. 10, article 133, 199.

[27] F. Hasenbalg, "Annual modulation sensitivity in cold dark matter searches," Astroparticle Physics, vol. 9, article 339, 1998.

[28] J. D. Vergados, "Theoretical directional and modulated rates for direct supersymmetric dark matter detection," Physical Review D: Particles, Fields, Gravitation and Cosmology, vol. 67, no. 10, 2003.

[29] A. M. Green, "Effect of realistic astrophysical inputs on the phase and shape of the WIMP annual modulation signal," Physical Review D: Particles, Fields, Gravitation and Cosmology, vol. 68, article 023004, 2004.
[30] C. Savage, K. Freese, and P. Gondolo, "Annual modulation of dark matter in the presence of streams," Physical Review D: Particles, Fields, Gravitation and Cosmology, vol. 74, no. 4, 2006.

[31] P. J. Fox, J. Kopp, M. Lisanti, and N. Weiner, "A CoGeNT modulation analysis," Physical Review D: Particles, Fields, Gravitation and Cosmology, vol. 85, no. 3, Article ID 036008, 2012.

[32] R. Essig, J. Mardon, and T. Volansky, "Direct detection of sub-GeV dark matter," Physical Review D: Particles, Fields, Gravitation and Cosmology, vol. 85, Article ID 076007, 2012.

[33] R. Essig, A. Manalaysay, J. Mardon, P. Sorensen, and T. Volansky, "First direct detection limits on Sub-GeV dark matter from XENON10," Physical Review Letters, vol. 109, no. 2, Article ID 021301, 2012.

[34] J. Vergados, H. Ejiri, and K. Savvidy, "Theoretical direct WIMP detection rates for transitions to excited states," Nuclear Physics $B$, vol. 877, article 36, 2013.

[35] J. Vergados, F. T. Avignone-III, M. Kortelainen et al., "Inelastic WIMP-nucleus scattering to the first excited state in ${ }^{125} \mathrm{Te}$," Journal of Physics G: Nuclear and Particle Physics, vol. 49, Article ID 150021, 2016.

[36] J. D. Vergados, "Searching for cold dark matter," Journal of Physics G: Nuclear and Particle Physics, vol. 22, no. 2, pp. 253272, 1996.

[37] M. Cannoni, "On the formalism and upper limits for spindependent cross sections in dark matter elastic scattering with nuclei," Physical Review D, vol. 84, article 095017, 2011.

[38] M. Cannoni, J. D. Vergados, and M. E. Gómez, "Scheme for the extraction of WIMP-nucleon scattering cross sections from total event rates," Physical Review D: Particles, Fields, Gravitation and Cosmology, vol. 83, no. 7, 2011.

[39] V. K. Oikonomou, J. D. Vergados, and C. C. Moustakidis, "Direct detection of dark matter rates for various wimps," Nuclear Physics B, vol. 773, no. 1-2, pp. 19-42, 2007.

[40] K. G. Savvidy and J. D. Vergados, "Direct dark matter detection: A spin," Physical Review D: Particles, Fields, Gravitation and Cosmology, vol. 87, article 075013, 2013.

[41] E. Aprile, K. Arisaka, F. Arneodo et al., "Dark Matter Results from 100 Live Days of XENON100 Data," Physical Review Letters, vol. 107, article 131302, 2011.

[42] E. Behnke, J. Behnke, S. J. Brice et al., "First dark matter search results from a 4 -kg CF3I bubble chamber operated in a deep underground site," Physical Review D, vol. 86, article 052001, 2012, see also Erratum: Phys. Rev. D 90, 079902 (2014).

[43] M. Felizardo, T. Morlat, A. C. Fernandes et al., "First Results of the Phase II SIMPLE Dark Matter Search," Physical Review Letters, vol. 108, article 201302, see also Erratum: Phys. Rev. D 90, 079902 (2014).

[44] S. Archambault, "Constraints on Low-Mass WIMP Interactions on 19F from PICASSO," Physics Letters B, vol. 711, article 153, 2012.

[45] S. Archambault, F. Aubin, and M. Auger, "Dark Matter Spin-Dependent Limits for WIMP Interactions on $19-\mathrm{F}$ by PICASSO," Physics Letters B, vol. 682, article 185, 2009.

[46] F. Aubin, M. Auger, and M.-H. Genest, "Discrimination of nuclear recoils from alpha particles with superheated liquids," New Journal of Physics, vol. 10, article 103017, Article ID 103017, 2008.

[47] E. Aprile, M. Alfonsi, K. Arisaka et al., "Dark Matter Results from 225 Live Days of XENON100 Data," Physical Review Letters, vol. 109, article 181301, Article ID 181301, 2012. 
[48] N. Abgrall, E. Aguayo, F. T. Avignone III et al., "The MAJORANA DEMONSTRATOR Neutrinoless Double-Beta Decay Experiment," Advances in High Energy Physics, vol. 2014, Article ID 365432, 18 pages, 2014.

[49] R. Agnese et al., "Silicon detector dark matter results from the final exposure of cdms II," Phys. Rev. Lett, vol. 111, Article ID 251301, 2013.

[50] J.-W. Chen, H.-C. Chi, and H.-B. Li, "Constraints on millicharged neutrinos via analysis of data from atomic ionizations with germanium detectors at sub-keV sensitivities," Physical Review D, vol. 90, article 011301(R), 2014.

[51] P. Venkataramaiah, K. Gopala, A. Basavaraju, S. S. Suryanarayana, and H. Sanjeeviah, "A simple relation for the Fermi function," Journal of Physics G: Nuclear and Particle Physics, vol. 11, no. 3, article no. 014, pp. 359-364, 1985.

[52] L. D. Landau and E. M. Lifshitz, Quantum Mechanics, Nonrelativistic Theory, Pergamon Press, 3rd edition, 1977.

[53] Y. Hochberg, M. Pyle, Y. Zhao, and K. M. Zurek, "Detecting superlight dark matter with Fermi-degenerate materials," Journal of High Energy Physics, vol. 2016, no. 8, article no. 57, 2016.

[54] K. Schutz and K. M. Zurec, "On the Detectability of Light Dark Matter with Superfluid Helium," Physical Review Letters, vol. 117, article 121302, 2016. 

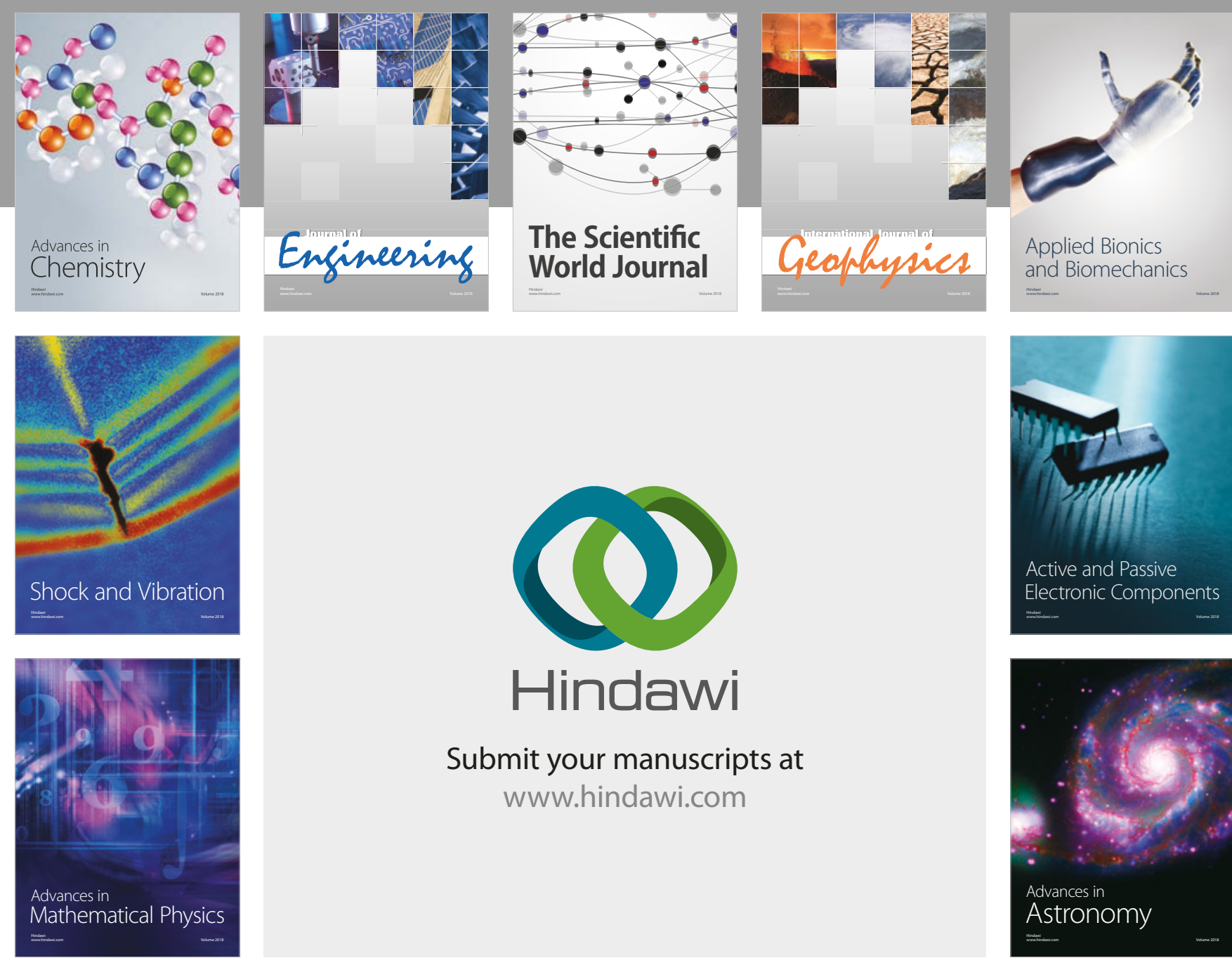

Submit your manuscripts at

www.hindawi.com

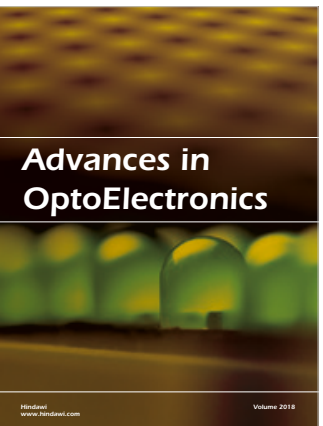

\section{Rotcting Machinery}
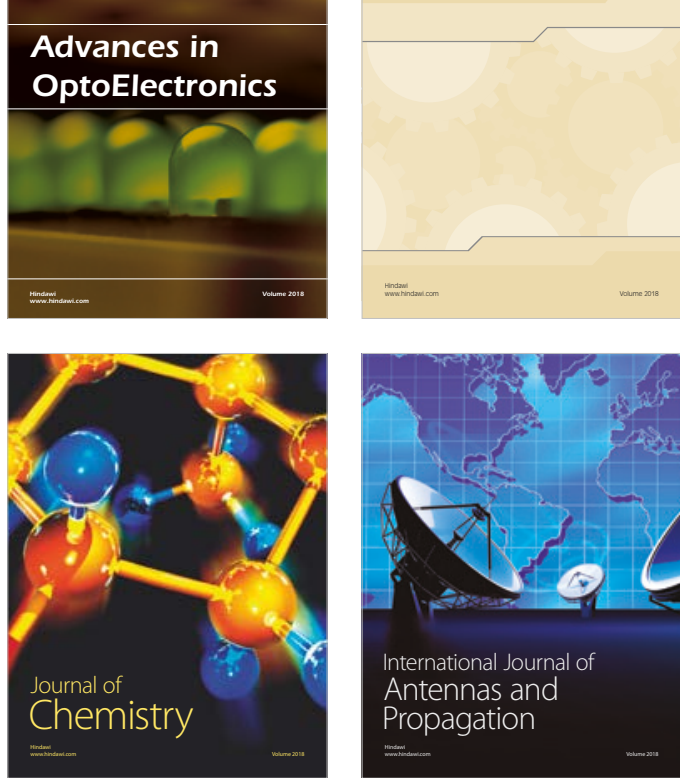

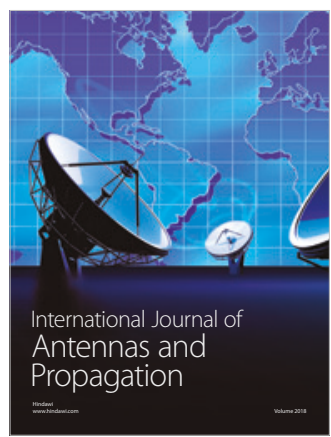

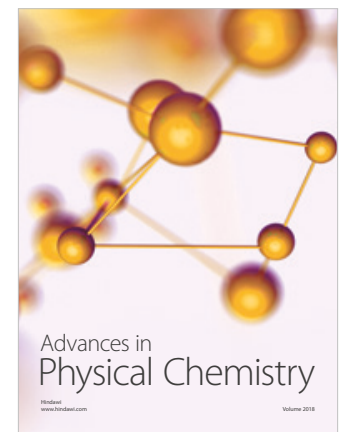

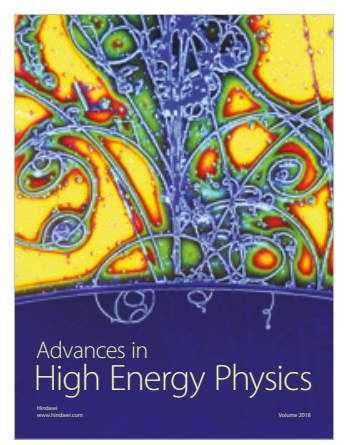

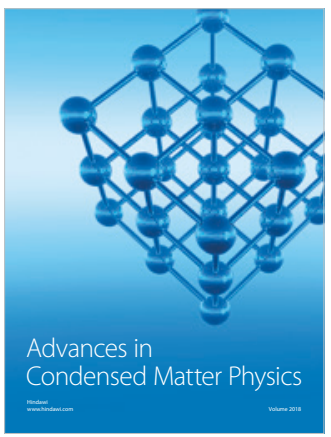

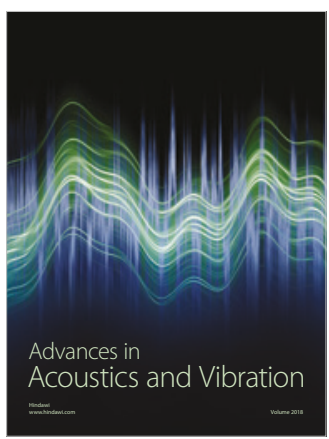

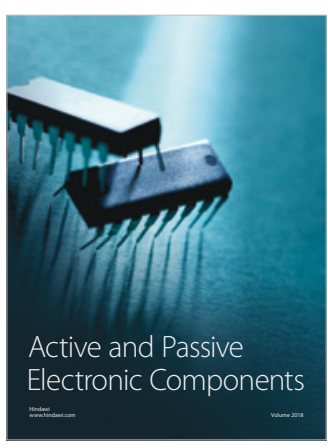
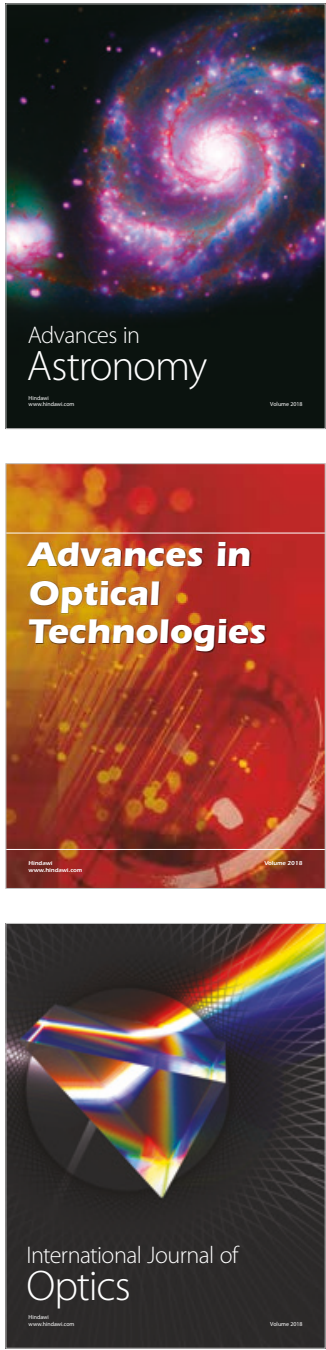